\title{
Biotyping of Haemophilus using API 10S-an epidemiological tool?
}

\author{
S MEHTAR, S AMINI AFSHAR \\ From the Department of Microbiology, North Middlesex Hospital, Edmonton, London N18 1QX
}

SUMMARY One hundred and ninety-nine strains of Haemophilus isolates were biotyped by Kilian'si் method $^{1}$ and a modified API $10 S$ strip and the results compared. One hundred percent correlation: was found between the two systems. The ONPG test proved of value in differentiating betweeno Haemophilus influenzae and Haemophilus parainfluenzae when there was growth factor discog failure.

Haemophilus influenzae a well-recognised pathogen, has been implicated in paediatric outbreaks particularly in the USA. ${ }^{23}$ The biotyping method described by Kilian has revived interest in Haemophilus as an organism involved in cross infection in hospitals and families. Capsular typing, though an important marker of invasiveness, has proved of limited value in such situations because of the heterogeneity of the Haemophilus population and the unreliability of the available typing antisera.

Biotyping has been found to be more consistent and we feel has proved to be a useful epidemiological tool.

Preparation of sugars in the laboratory by the described conventional method was tedious and timeconsuming. API $10 \mathrm{~S}$ strip contained the relevant biochemical tests and the suspending medium was modified to support growth of haemophili without interfering with results.

\section{Material and methods}

\section{ISOLATES}

All clinical isolates of Haemophilus identified in the laboratory between December 1981 and February 1982 were examined. Haemophilus was identified by picking off suspicious colonies from a chocolate agar plate (containing 10 units of bacitracin) after $18 \mathrm{~h}$ incubation in $\mathrm{CO}_{2}$ at $37^{\circ} \mathrm{C}$. Gram strain, growth dependence on " $X$ " and " $V$ ", purity plate and antibiotic disc sensitivity testing were performed on a single colony suspended in ordinary peptone water. The purity plates were then used for biotyping.

Accepted for publication 16 July 1982
Growth dependence and antibiograms were repeatef and biochemical profiles determined by both methods? Pittman serotyping using Wellcome's antisera a-f wast performed on $H$ influenzae strains.

\section{BIOTYPING}

Kilian's method was used to prepare biochemica tests in the laboratory as described.' Four biog chemical tests, urea, ornithine, indole and $\beta$-galactõ sidase (ONPG) were selected which adequatel covered the identification of both $H$ influenzae and $F_{0}$ parainfluenzae.

API $10 S$ strips were found to contain the same selection of biochemical tests required. After a pilo study using glucose broth, and API water supple mented with $10 \% \mathrm{X}$ and $\mathrm{V}$, supplemented peptone water was found to be the most suitable and was prepared as follows:

(a) Peptone water (Southern Group) commercial available in $3 \mathrm{ml}$ quantities.

(b) $N A D(B D H)$ made up according to the manufacturer's instructions. Distilled water $(5 \mathrm{~m}$ was added to each vial containing $25 \mathrm{mg}$ of NAD; 0.3. $\mathrm{ml}$ of this was added to peptone water.

(c) Haemin $1 \%(\mathrm{BDH})$ when reconstituted, was sufficient for over 3000 tests; $0 \cdot 3 \mathrm{ml}$ was added to the peptone water.

One colony of Haemophilus was suspended in ordinary peptone water, and the NAD and Haemi added after growth requirements and antibiogram 8 had been performed. The API $10 \mathrm{~S}$ was then inoculated. The indole microtube in the strip was overlaid with sterile mineral oil along with the ureat and ornithine. Saline containing one colony of Haemophilus was used as a suspending medium to inoculate the conventional sugars. Both sets of sugars 
were incubated at $37^{\circ} \mathrm{C}$ for $18 \mathrm{~h}$ aerobically. The next morning oil from the indole microtube in the API $10 \mathrm{~S}$ strip was removed with a pipette and Kovac's reagent was added. Kovac's reagent was also used for detection of indole in the conventional sugars.

Known NCTC strains of haemophili, $H$ influenzae biotypes I-V and $H$ parainfluenzae biotypes I-III were used as control strains.

\section{Results}

A total of 199 strains of $H$ influenzae were tested for biochemical reactions and Kilian's method and API $10 \mathrm{~S}$ compared. Using growth requirements, 105 strains were identified as $H$ influenzae and 94 as $H$ parainfluenzae in the routine laboratory. Biotyping of these strains identified $105 \mathrm{H}$ influenzae, $72 \mathrm{H}$ parainfluenzae, $20 \mathrm{H}$ para-aphrophilus (ONPGpositive only) and two non-typable strains (indole and ONPG-positive). The biochemical identification for each biotype of $H$ influenzae and $H$ parainfluenzae is shown in Table 1.

There was a $100 \%$ correlation between the two methods used. $H$ influenzae biotypes $\mathrm{I}$ to $\mathrm{V}$ were easily identified by their lack of $\dot{\beta}$-galactosidase. In contrast, $H$ parainfluenzae strains were ONPG-positive in all but three cases: one strain belonged to biotype I and there were two biotype III strains. Twenty strains gave no reactions to urea, indole or ornithine in either system but were ONPG-positive (Table 1). These strains were non-haemolytic and "V" dependent and therefore were biochemically classed as $H$ parainfluenzae. Two non-typable strains were indole-positive as well as being ONPG-positive. We found no ONPG-positive " $X$ \& V" dependent strains. ONPG was a good differential test between $H$ influenzae and $H$ parainfluenzae where there was growth factor disc failure and gave a $98.4 \%$ positive identification for $H$ parainfluenzae in both systems (Table 1).

Table 1 Biochemical profile of 199 isolates of Haemophilus

\begin{tabular}{rllll}
\hline & $\begin{array}{l}\text { API/CS } \\
\text { Urea }\end{array}$ & $\begin{array}{l}\text { API/CS } \\
\text { Ornithine }\end{array}$ & $\begin{array}{l}\text { API/CS } \\
\text { Indole }\end{array}$ & $\begin{array}{l}\text { API/CS } \\
\text { ONPG }\end{array}$ \\
\hline Hinfluenzae & Total 105 & & & \\
Biotype I 19 & $19 / 19$ & $19 / 19$ & $19 / 19$ & $0 / 0$ \\
II 57 & $57 / 57$ & $0 / 0$ & $57 / 57$ & $0 / 0$ \\
III 21 & $21 / 21$ & $0 / 0$ & $0 / 0$ & $0 / 0$ \\
IV 6 & $6 / 6$ & $6 / 6$ & $0 / 0$ & $0 / 0$ \\
V 2 & $0 / 0$ & $2 / 2$ & $2 / 2$ & $0 / 0$ \\
H parainfluenzae & Total 72 & & & \\
Biotype I 29 & $0 / 0$ & $29 / 29$ & $0 / 0$ & $28 / 28$ \\
II 28 & $28 / 28$ & $28 / 28$ & $0 / 0$ & $28 / 28$ \\
III 15 & $15 / 15$ & $0 / 0$ & $0 / 0$ & $13 / 13$ \\
Haphrophilus & Total 20 & & & \\
20 & $0 / 0$ & $0 / 0$ & $0 / 0$ & $20 / 20$ \\
Non-typable 2 & $0 / 0$ & $0 / 0$ & $2 / 2$ & $2 / 2$ \\
\hline
\end{tabular}

CS = Kilian's method.

API = API 10S strip.
DISTRIBUTION OF BIOTYPES

Amongst $H$ influenzae, biotype II was the commonest. Of the 150 strains biotyped, biotype I was $18 \cdot 2 \%$, biotype II $54.8 \%$, biotype III $20 \cdot 1 \%$, biotype IV $5.7 \%$ and biotype V $1.9 \%$. The $72 \mathrm{H}$ parainfluenzae strains were $40 \cdot 2 \%, 38.8 \%$ and $20 \%$ respectively in biotypes I, II, III. H para-aphrophilus made up of $10 \%$ of the total number of haemophili isolated; there were only two non-typable strains. Thirty-four $(32.3 \%)$ capsulated strains of $H$ influenzae were found. These were mainly amongst biotypes I and II: the commonest being Pittman type b (Table 2).

Twenty-one of the 34 strains belonged to biotype II $(36.8 \%)$ and to biotype I ( $42 \%)$. We feel the number of capsulated strains would have been higher but the agglutination method is limited by the available antisera and the selection of single colonies from the original plates for purity.

\section{CORRELATION WITH ISOLATION SITES}

Haemophilus influenzae was isolated from sputum, CSF, blood, conjunctiva, nose, throat, antral washouts (Table 3 ). In contrast, $H$ parainfluenzae strains were restricted to the respiratory tract and were repeatedly isolated from purulent and mucopurulent sputum from patients with obvious chest infections where no other pathogens were found. Some strains of $H$ para-aphrophilus were found mixed with $H$ influenzae strains and were regarded as commensals. One hundred and eightyfive of 199 strains were isolated from the respiratory tract $(92 \%), 2.5 \%$ from conjunctiva and $1.5 \%$ from CSF. $H$ influenzae strains found in sputum belonged mainly to biotypes I and II. CSF isolates belonged to biotype I but only one of them was capsulated. This strain was isolated from the blood as well. The $H$ influenzae biotype II was isolated from the CSF of a 55-year-old woman and was non-capsulated. Isolates from the conjunctiva belonged only to biotypes II and III of $H$ influenzae. The association of various biotypes of $H$ influenzae and $H$ parainfluenzae associated with antibiotic resistance will be published separately.

Table 2 Capsular type distribution amongst the various biotypes of $H$ influenzae

\begin{tabular}{ccccccc}
\hline \multicolumn{7}{c}{ Pittman type } \\
\cline { 2 - 7 } Biotype & $a$ & $b$ & $c$ & $d$ & $e$ & $f$ \\
\hline I (19) & 1 & 6 & 0 & 1 & 0 & 0 \\
II (57) & 9 & 7 & 4 & 1 & 0 & 0 \\
III (21) & 0 & 2 & 3 & 0 & 0 & 0 \\
IV (6) & 0 & 0 & 0 & 0 & 0 & 0 \\
V (2) & 0 & 0 & 0 & 0 & 0 & 0 \\
Total (34) & 10 & 15 & 7 & 2 & 0 & 0 \\
\hline
\end{tabular}


Table 3 Distribution of various biotypes from clinical specimens

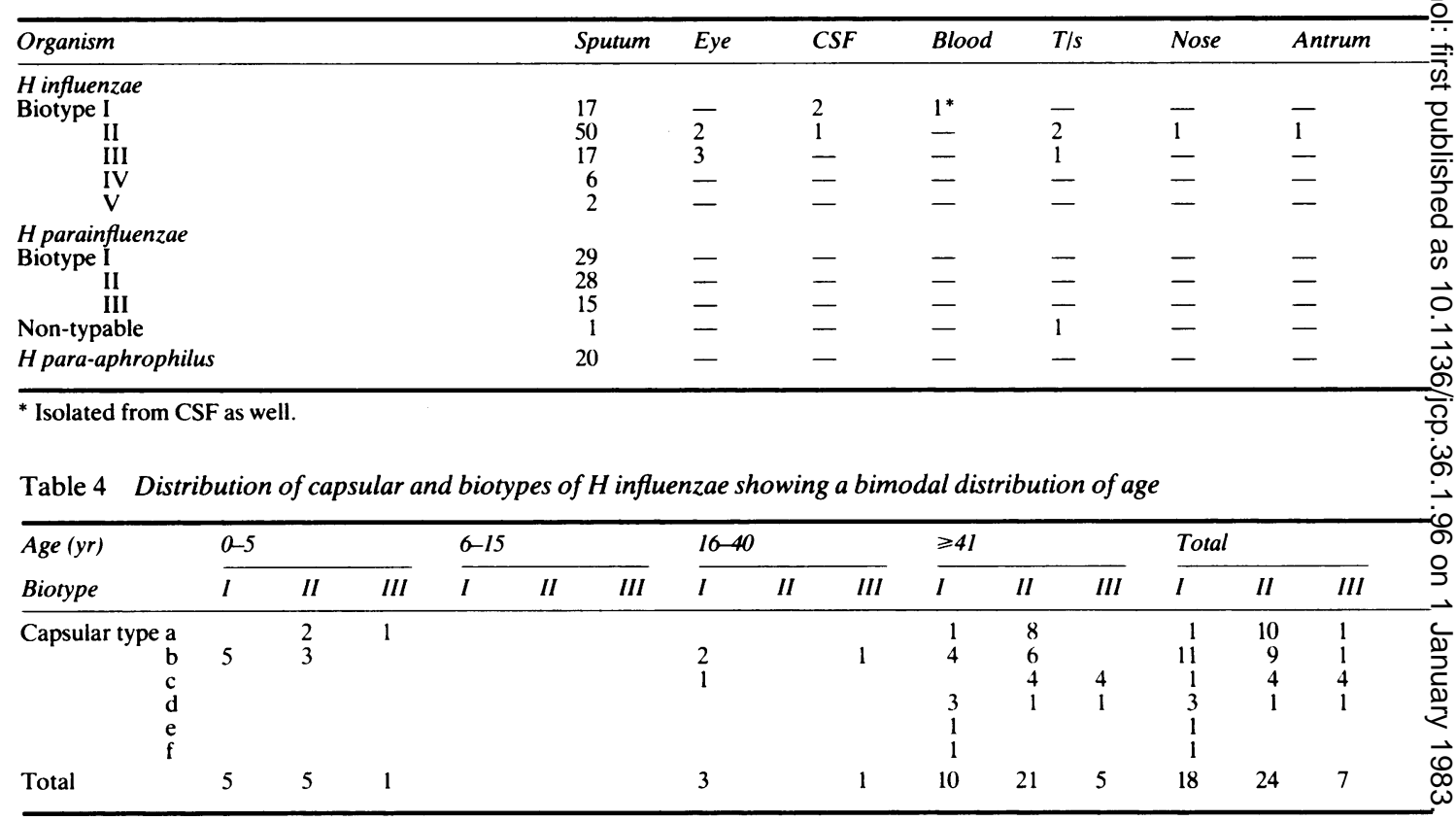

The age distribution in relation to various biotypes and serotypes is given in Table $4 . H$ influenzae showed a biphasic distribution between the ages of $0-5 \mathrm{yr}$ and $>40 \mathrm{yr}$. The isolates from patients below 5 yr of age were isolated from CSF, blood and conjunctiva whereas those above the age of $40 \mathrm{yr}$ were mainly from the respiratory tract with one from CSF, another from an antral wash out and one from the eye. Below the age of five, biotypes I and II were equally distributed and capsular type $b$ was commonest. After the age of $40 \mathrm{yr}$, most of the isolates were from the respiratory tract. Even though biotype II was predominant amongst $H$ influenzae, capsular serotypes "a" and "b" were evenly distributed with types "c" and " $d$ " being the next commonest. The other two biotypes of $H$ influenzae - that is, IV and V, were found to be noncapsulated.

\section{Discussion}

Biotyping of haemophili is a reproducible method of epidemiological tracing. Capsular typing poses problems of a heterogeneous population of $H$ influenzae and is also limited by the agglutination results with the available antisera. The selection of single colonies of the various biotypes may account for the $30 \%$ of capsulated strains found in this study; had twenty or more colonies been tested, possibly a higher incidence of capsulated strains would have been found.

Other rapid methods such as Micro $\mathrm{ID}^{4}$ and the्D Minitek $^{5}$ have been used as a rapid biotyping method and compared with Kilian's method. The Micro IDP gave $100 \%$ correlation except with ornithine which was $96 \%$. The Minitek was less favourable with of $97.5 \%$ correlation in the indole test and $92.3 \%$ in the ornithine. However, using the API 10S strip a $100 \%$ correlation in all four tests was achieved wherg compared with Kilian.

The ONPG test proved helpful in differentiating $H_{\mathfrak{B}}$ influenzae (ONPG negative) from $H$ parainfluenza particularly when there was diffusion of growthg factors through the agar; $98.4 \%$ of $H$ parainfluenzaes were ONPG + ve; ONPG + ve "V" dependent strains? that did not utilise substrates were classified as $h$ para-aphrophilus by Kilian. However, Oberhoffer? and Back $^{6}$ reasonably suggest these be included in $H_{5}$ parainfluenzae as biotype IV. $\beta$-galactosidase-posi tive strains which are also indole-positive but " $V$ '? dependent were considered non-typable.

Welch et $a l^{7}$ have reported a higher incidence of indole-producing $H$ influenzae from CSF than the respiratory tract, thus pointing out the relevance of $H \mathrm{O}$ influenzae biotype I and II in meningitis. Therefore an indole and ONPG positive strain is unlikely to be $H$ influenzae and we feel should be classified $\vec{D}$ separately. 
The capsulated strains found under the age of $5 \mathrm{yr}$ made up approximately half the total $H$ influenzae isolates. It is therefore possible that serious infection can be caused by non-capsulated strains of $H$ influenzae in the young.

After the age of $40 \mathrm{yr}$ the incidence of $H$ influenzae increased, but only a third of these strains was capsulated. This might reflect the lack of or loss of immunity in these patients. Long term colonisation of chronic bronchitis can cause the loss or gain of capsules and a mixed population of Haemophilus. The administration of antibiotics in acute exacerbation of chronic bronchitis could possibly induce resistant strains of other species to transfer plasmids to haemophili regardless of their capsules. Antibiotic resistance and the transfer of plasmids between similar or different biotypes of $H$ influenzae and $H$ parainfluenzae is becoming increasingly important. Capsular typing, a marker for virulence, may fall short when multiresistant non-capsulated strains of $H$ influenzae produce severe systemic infection or where there may be cross infection. ${ }^{8} \mathrm{We}$ feel that using the API 10S, biotyping is easy, reliable and rapid and can be a useful adjunct to the existing methods in the identification of haemophili.

\section{References}

' Kilian M. A taxonomic study of the genus Haemophilus with the proposal of a new species. J Glen Microbiol 1976;93:9-62.

${ }^{2}$ Glode MP, Schiffer MS, Robbins JB, et al. An outbreak of Haemophilus influenzae type B meningitis in an enclosed hospital population. J Pediatr 1976;88:36-40.

${ }^{3}$ Ginsburg CM, McCracken GH, Roe S, Park JG. Haemophilus influenzae type $\mathrm{B}$ disease: incidence in a day centre. JAMA 1977;238:604-7.

${ }^{4}$ Back AE, Oberhoffer TR. Use of the Minitek system for biotyping Haemophilus species. J Clin Microbiol 1978;7:312-3.

${ }^{5}$ Edberg CS, Melton E, Singer JM. Rapid biochemical characterization of Haemophilus species by using Micro-ID. J Clin Microbiol 1980;11:22-6.

- Oberhoffer TR, Back AE. Biotypes of Haemophilus encountered in clinical laboratories. J Clin Microbiol 1979;10:168-74.

7 Welch DF, Ahlia PA, Matsen JM. Differentiation of Haemophilus species in respiratory isolates cultures by an indole spot test. J Clin Microbiol 1982;2:216-9.

${ }^{8}$ Mehtar S, Law C. Outbreak of $\beta$-lactamase producing Haemophilus influenzae (biotype III) in a geriatric ward. J Hosp Infect 1980;1:357-8.

Requests for reprints to: Dr S Mehtar, Department of Microbiology, North Middlesex Hospital, Edmonton, London N18 1QX, England. 